\title{
Exact One-Point Function of $N=1$ super-Liouville Theory with Boundary
}

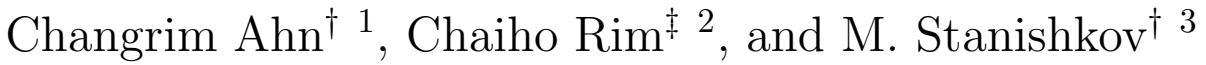 \\ †Department of Physics, Ewha Womans University \\ Seoul 120-750, Korea \\ $\ddagger$ Department of Physics, Chonbuk National University \\ Chonju 561-756, Korea
}

PACS: 11.25.Hf, 11.55.Ds

\begin{abstract}
In this paper, exact one-point functions of $N=1$ super-Liouville field theory in two-dimensional space-time with appropriate boundary conditions are presented. Exact results are derived both for the theory defined on a pseudosphere with discrete (NS) boundary conditions and for the theory with explicit boundary actions which preserves super conformal symmetries. We provide various consistency checks. We also show that these one-point functions can be related to a generalized Cardy conditions along with corresponding modular $S$-matrices. Using this result, we conjecture the dependence of the boundary two-point functions of the (NS) boundary operators on the boundary parameter.
\end{abstract}

\footnotetext{
${ }^{1}$ ahn@dante.ewha.ac.kr

${ }^{2}$ rim@phy.chonbuk.ac.kr

${ }^{3}$ stanishkov@dante.ewha.ac.kr; On leave of absence from Institute of Nuclear Research and Nuclear Energy, Sofia, Bulgaria
} 


\section{Introduction}

Liouville field theory (LFT) has been studied actively for its relevance with non-critical string theories and two-dimensional quantum gravity. This theory is also interesting on its own as an example of irrational conformal field theory (CFT). Most CFT formalism developed for rational CFTs do not apply to this class of theories mainly because they have continuously infinite number of primary fields. Various methods have been proposed to derive structure constants and reflection amplitudes, which are basic building blocks to complete the conformal bootstrap [1, 2, 3].

Another example of the irrational CFT is the $N=1$ supersymmetric LFT (SLFT). This model has some motivations. It is applicable to the superstring theories and the two-dimensional supergravity with fermionic matter fields. One can also understand the role of the extended conformal symmetry in the irrational CFTs by studing this model. The methods introduced for the bulk LFT have been applied successfully to the bulk SLFT although the latter becomes algebraically more complicated [4, 5].

It is interesting to extend these formalisms to the CFTs defined in the two-dimensional space-time geometry with a boundary condition (BC) which preserves the conformal symmetry. Cardy showed that the conformally invariant BCs can be associated with the primary fields in terms of modular $S$-matrix elements for the case of rational CFTs [6]. It has been an issue whether the Cardy formalism can be extended to the irrational CFTs. Another motiviation is to understand open string theories in various nontrivial background space-time geometries [7].

Recently it has been shown that functional relation method developed in [2] can be used in the boundary LFT with conformal boundary action to derive one-point function of a bulk operator and correlation functions of two boundary operators for a given conformal $\mathrm{BC}$ [8]. Here the conformal $\mathrm{BC}$ is denoted by a continuous parameter related to the coupling constant in the boundary action. Another development made in [9] is to generalize this method to the boundary LFT defined in the classical Lobachevskiy plane, namely the pseudosphere. These works showed that the one-point functions of primary fields can be related to the inner products of the conformal BCs and different Ishibashi states in the same way as the rational CFTs [10].

It has been noticed that generalizing the Cardy formalism to the supersymmetric CFTs is nontrivial even for rational theories mainly because the Ramond (R) sector transforms not to Neveu-Schwarz (NS) sector but so-called (NS) sector under the modular transformation [11, 12]. In this paper we show that it is possible to derive exact onepoint functions from the functional relations and relate these to the Cardy formalism for the BCs introduced in the pseudosphere and ordinary half plane with conformally invariant boundary action. This shows that the Cardy formalism can be generalized to the irrational super-CFTs. We also show that the boundary two-point functions of the (NS) boundary operators satisfy the same relation as those of the LFT. This paper is organized as follows. In sect. 2 we compute the one-point functions of the SLFT primary 
fields on the pseudosphere. In sect.3 we propose the boundary action which preserves the superconformal symmetry and compute the corresponding one-point functions. These results are used in sect. 4 to be related to the conformal BCs and are shown to be consistent with the Cardy formalism. We conclude the paper with some comments in sect.5.

\section{One-Point Functions of SLFT on a Pseudosphere}

The $N=1$ SLFT describes a supermultiplet consisting of a bosonic field and its fermionic partner interacting with exponential potential. In terms of the component fields, the Lagrangian can be expressed by

$$
\mathcal{L}_{\mathrm{SL}}=\frac{1}{8 \pi}\left(\partial_{a} \phi\right)^{2}-\frac{1}{2 \pi}(\bar{\psi} \partial \bar{\psi}+\psi \bar{\partial} \psi)+i \mu b^{2} \psi \bar{\psi} e^{b \phi}+\frac{\pi \mu^{2} b^{2}}{2}\left(: e^{b \phi}:\right)^{2} .
$$

The first interaction term in Eq.(1) containing $\exp (b \phi)$ has (holomorphic) conformal dimension $1 / 2$. This is correct since the Majorana fermion field has dimension $1 / 2$. The SLFT is a superconformal field theory. With the background charge $Q$

$$
Q=b+\frac{1}{b}
$$

the SLFT has the central charge

$$
c_{S L}=\frac{3}{2}\left(1+2 Q^{2}\right)
$$

The bulk (NS) and (R) primary fields of the SLFT are given by

$$
N_{\alpha}(z, \bar{z})=e^{\alpha \phi}, \quad R_{\alpha}(z, \bar{z})=\sigma^{(\epsilon)}(z, \bar{z}) e^{\alpha \phi},
$$

where $\sigma^{( \pm)}$is the 'spin field' with dimension 1/16 with even Fermi number for $(+)$and odd for $(-)$ and satisfying an operator product expansion (OPE) $\sigma^{(+)} \sigma^{(-)}=\psi$. f The conformal dimensions become, respectively,

$$
\Delta_{\alpha}^{N}=\frac{1}{2} \alpha(Q-\alpha), \quad \Delta_{\alpha}^{R}=\frac{1}{16}+\frac{1}{2} \alpha(Q-\alpha)
$$

From these, one can see that physical states can be denoted by a real parameter $P$ defined by

$$
\alpha=\frac{Q}{2}+i P
$$

In this section we are interested in the SLFT on a pseudosphere. This is a generalization of [9] where the LFT is studied in the geometry of the infinite constant negative

\footnotetext{
${ }^{4}$ We will suppress the superindex $(\epsilon)$ as much as we can since the correlation functions of our interest do not depend on it explicitly as we will show later.
} 
curvature surface, the so-called Lobachevskiy plane, i.e. the pseudosphere. The equations of motion for the SLFT are given by

$$
\begin{aligned}
\partial \bar{\partial} \phi & =4 \pi^{2} \mu b^{2}\left(\mu e^{b \phi}-i \bar{\psi} \psi\right) e^{b \phi} \\
\partial \bar{\psi} & =-i \mu e^{b \phi} \psi, \quad \bar{\partial} \psi=i \mu e^{b \phi} \bar{\psi}
\end{aligned}
$$

We will assume that the fermion vanishes in the classical limit so that the background metric is determined by the bosonic field satisfying

$$
e^{\varphi(z)}=\frac{4 R^{2}}{\left(1-|z|^{2}\right)^{2}},
$$

where $\varphi=2 b \phi$ and $R^{-2}=4 \pi^{2} \mu^{2} b^{3}$. The parameter $R$ is interpreted as the radius of the pseudosphere in which the points at the circle $|z|=1$ are infinitely far away from any internal point. This circle can be interpreted as the "boundary" of the pseudosphere. In the same way as the LFT, we can now use the Poincaré model of the Lobachevskiy plane with complex coordinate $\xi$ in the upper half plane.

We want to compute exact one-point functions of the (NS) and (R) bulk operators $N_{\alpha}$ and $R_{\alpha}$. Due to the superconformal invariance, these one-point functions are given by

$$
\begin{aligned}
\left\langle N_{\alpha}(\xi)\right\rangle & =\frac{U^{N}(\alpha)}{|\xi-\bar{\xi}|^{2 \Delta_{\alpha}^{N}}} \\
\left\langle R_{\alpha}(\xi)\right\rangle & =\frac{U^{R}(\alpha)}{|\xi-\bar{\xi}|^{2 \Delta_{\alpha}^{R}}} .
\end{aligned}
$$

We will simply refer to the coefficients $U^{N}(\alpha)$ and $U^{R}(\alpha)$ as bulk one-point functions. To derive the functional relations satisfied by these one-point functions, we should consider the bulk degenerate fields which are defined by some differential equations with certain orders [13].

The degenerate fields in the (NS) sector are given by

$$
N_{\alpha_{m, n}}=e^{\alpha_{m, n} \phi}, \quad \alpha_{m, n}=\frac{1}{2 b}(1-m)+\frac{b}{2}(1-n), \quad \text { with } \quad m-n=\text { even }
$$

and those in the $(\mathrm{R})$ sector by

$$
R_{\alpha_{m, n}}=\sigma^{(\epsilon)} e^{\alpha_{m, n} \phi}, \text { with } m-n=\text { odd. }
$$

One of the essential features of these fields is that the operator product expansion (OPE) of a degenerate field with any primary field is given by a linear combination of only finite number of primary fields and their decendents. The simplest degenerate fields are $N_{-b}$ for the (NS) sector and $R_{-b / 2}$ for the $(\mathrm{R})$ sector. In particular, $R_{-b / 2}$ satisfies

$$
\left[\frac{1}{b^{2}} \partial+G_{-1} G_{0}\right] R_{-b / 2}=0
$$


where $G$ is the fermionic part of the supercurrent. Similar equation holds for the antiholomorphic part.

The OPE of $R_{-b / 2}$ with a (NS) primary field is given by

$$
N_{\alpha} R_{-b / 2}=C_{+}^{(N)}(\alpha)\left[R_{\alpha-b / 2}\right]+C_{-}^{(N)}(\alpha)\left[R_{\alpha+b / 2}\right]
$$

where [...] stands for entire family of conformal decendents corresponding to a primary field. The structure constants can be computed using Coulomb gas integrals. One can set $C_{+}^{(N)}=1$ since no screening insertion is needed. The other structure constant $C_{-}^{(N)}$ needs just one insertion of the SLFT interaction and can be obtained by

$$
\begin{aligned}
C_{-}^{(N)}(\alpha) & =-i \mu b^{2} \int d^{2} \xi\left\langle N_{\alpha}(0) R_{-b / 2}(1) \psi(\xi) \bar{\psi}(\bar{\xi}) e^{b \phi(\xi, \bar{\xi})} R_{Q-\alpha-b / 2}(\infty)\right\rangle \\
& =\frac{\mu b^{2}}{2} \int d^{2} \xi|\xi|^{-2 \alpha b}|1-\xi|^{b^{2}-1}=\frac{\pi \mu b^{2} \gamma\left(\alpha b-\frac{b^{2}}{2}-\frac{1}{2}\right)}{2 \gamma\left(\frac{1-b^{2}}{2}\right) \gamma(\alpha b)}
\end{aligned}
$$

with $\gamma(x)=\Gamma(x) / \Gamma(1-x)$. Here, we used the following correlation functions of the spin fields

$$
\begin{aligned}
\left\langle\sigma\left(\xi_{1}, \bar{\xi}_{1}\right) \sigma\left(\xi_{2}, \bar{\xi}_{2}\right)\right\rangle & =\frac{1}{\left|\xi_{12}\right|^{1 / 4}} \\
\left\langle\sigma\left(\xi_{1}, \bar{\xi}_{1}\right) \sigma\left(\xi_{2}, \bar{\xi}_{2}\right) \psi\left(\xi_{3}\right) \bar{\psi}\left(\bar{\xi}_{3}\right)\right\rangle & =\frac{i / 2}{\left|\xi_{12}\right|^{-3 / 4}\left|\xi_{13}\right|\left|\xi_{23}\right|}
\end{aligned}
$$

Similarly, the OPE with the $(\mathrm{R})$ primary field is

$$
R_{\alpha} R_{-b / 2}=C_{+}^{(R)}(\alpha)\left[N_{\alpha-b / 2}\right]+C_{-}^{(R)}(\alpha)\left[N_{\alpha+b / 2}\right]
$$

where $C_{+}^{(R)}=1$ as before and $C_{-}^{(R)}$ is given by a screening integral

$$
\begin{aligned}
C_{-}^{(R)}(\alpha) & =-i \mu b^{2} \int d^{2} \xi\left\langle R_{\alpha}(0) R_{-b / 2}(1) \psi(\xi) \bar{\psi}(\bar{\xi}) e^{b \phi(\xi, \bar{\xi})} N_{Q-\alpha-b / 2}(\infty)\right\rangle \\
& =\frac{\mu}{2} b^{2} \int d^{2} \xi|\xi|^{-2 \alpha b-1}|1-\xi|^{b^{2}-1}=\frac{\mu}{2} b^{2} \frac{\pi \gamma\left(\alpha b-\frac{b^{2}}{2}\right)}{\gamma\left(\frac{1-b^{2}}{2}\right) \gamma\left(\alpha b+\frac{1}{2}\right)}
\end{aligned}
$$

Now we consider the bulk two-point functions of the degenerate field $R_{-b / 2}$ and a (NS) field $N_{\alpha}$,

$$
G_{\alpha,-b / 2}^{N}\left(\xi, \xi^{\prime}\right)=\left\langle N_{\alpha}\left(\xi^{\prime}\right) R_{-b / 2}(\xi)\right\rangle
$$

It is straightforward from Eq.(15) to show that the two-point function satisfy

$$
G_{\alpha,-b / 2}^{N}\left(\xi, \xi^{\prime}\right)=C_{+}^{(N)}(\alpha) U^{R}\left(\alpha-\frac{b}{2}\right) \mathcal{G}_{+}\left(\xi, \xi^{\prime}\right)+C_{-}^{(N)}(\alpha) U^{R}\left(\alpha+\frac{b}{2}\right) \mathcal{G}_{-}\left(\xi, \xi^{\prime}\right)
$$


where $\mathcal{G}_{ \pm}\left(\xi, \xi^{\prime}\right)$ are expressed in terms of the special conformal blocks

$$
\mathcal{G}_{ \pm}\left(\xi, \xi^{\prime}\right)=\frac{\left|\xi^{\prime}-\bar{\xi}^{\prime}\right|^{2 \Delta_{\alpha}^{N}-2 \Delta_{-b / 2}^{R}}}{\left|\xi-\bar{\xi}^{\prime}\right|^{4 \Delta_{\alpha}^{N}}} \mathcal{F}_{ \pm}(\eta)
$$

with

$$
\eta=\frac{\left(\xi-\xi^{\prime}\right)\left(\bar{\xi}-\bar{\xi}^{\prime}\right)}{\left(\xi-\bar{\xi}^{\prime}\right)\left(\bar{\xi}-\xi^{\prime}\right)}
$$

Here, the conformal blocks are given by the hypergeometric functions

$$
\begin{aligned}
& \mathcal{F}_{+}(\eta)=\eta^{\frac{\alpha b}{2}}(1-\eta)^{-\frac{b^{2}}{4}+\frac{3}{8}} F\left(\alpha b-b^{2}, \frac{1}{2}-\frac{b^{2}}{2} ; \alpha b-\frac{b^{2}}{2}+\frac{1}{2} ; \eta\right), \\
& \mathcal{F}_{-}(\eta)=\eta^{\frac{1}{2}+\frac{b^{2}}{2}-\frac{\alpha b}{2}}(1-\eta)^{-\frac{b^{2}}{4}+\frac{3}{8}} F\left(\frac{1}{2}-\frac{b^{2}}{2}, 1-\alpha b ;-\alpha b+\frac{b^{2}}{2}+\frac{3}{2} ; \eta\right) .
\end{aligned}
$$

In the cross channel, an equivalent expression for the two-point function can be obtained as follows:

$$
G_{\alpha,-b / 2}^{N}=\frac{\left|\xi^{\prime}-\bar{\xi}^{\prime}\right|^{2 \Delta_{\alpha}^{N}-2 \Delta_{-b / 2}^{R}}}{\left|\xi-\bar{\xi}^{\prime}\right|^{4 \Delta_{\alpha}^{N}}}\left[B_{+}^{(N)}(\alpha) \widetilde{\mathcal{F}}_{+}(\eta)+B_{-}^{(N)}(\alpha) \widetilde{\mathcal{F}}_{-}(\eta)\right]
$$

with

$$
\begin{aligned}
& \widetilde{\mathcal{F}}_{+}(\eta)=\eta^{\frac{\alpha b}{2}}(1-\eta)^{-\frac{b^{2}}{4}+\frac{3}{8}} F\left(\alpha b-b^{2}, \frac{1}{2}-\frac{b^{2}}{2} ; 1-b^{2} ; 1-\eta\right), \\
& \widetilde{\mathcal{F}}_{-}(\eta)=\eta^{\frac{\alpha b}{2}}(1-\eta)^{\frac{3 b^{2}}{4}+\frac{3}{8}} F\left(\frac{1}{2}+\frac{b^{2}}{2}, \alpha b ; 1+b^{2} ; 1-\eta\right) .
\end{aligned}
$$

The boundary structure constants $B_{ \pm}^{(N)}$ can be determined from the monodromy relations

$$
\begin{aligned}
& \mathcal{F}_{+}(\eta)=\frac{\Gamma\left(\alpha b-\frac{b^{2}}{2}+\frac{1}{2}\right) \Gamma\left(b^{2}\right)}{\Gamma\left(\frac{b^{2}}{2}+\frac{1}{2}\right) \Gamma(\alpha b)} \widetilde{\mathcal{F}}_{+}(\eta)+\frac{\Gamma\left(\alpha b-\frac{b^{2}}{2}+\frac{1}{2}\right) \Gamma\left(-b^{2}\right)}{\Gamma\left(\alpha b-b^{2}\right) \Gamma\left(\frac{1}{2}-\frac{b^{2}}{2}\right)} \widetilde{\mathcal{F}}_{-}(\eta), \\
& \mathcal{F}_{-}(\eta)=\frac{\Gamma\left(-\alpha b+\frac{b^{2}}{2}+\frac{3}{2}\right) \Gamma\left(b^{2}\right)}{\Gamma\left(\frac{b^{2}}{2}+\frac{1}{2}\right) \Gamma\left(1+b^{2}-\alpha b\right)} \widetilde{\mathcal{F}}_{+}(\eta)+\frac{\Gamma\left(-\alpha b+\frac{b^{2}}{2}+\frac{3}{2}\right) \Gamma\left(-b^{2}\right)}{\Gamma(1-\alpha b) \Gamma\left(\frac{1}{2}-\frac{b^{2}}{2}\right)} \widetilde{\mathcal{F}}_{-}(\eta) .
\end{aligned}
$$

The conformal block $\widetilde{\mathcal{F}}$ - corresponds to the identity boundary operator with dimension 0 appearing in the boundary as the bulk operator $R_{-b / 2}$ approaches the boundary with $\eta \rightarrow 1$. Another boundary operator $n_{-b}$ appearing as $R_{-b / 2}$ approaches the boundary generates the $\widetilde{\mathcal{F}_{+}}$block. As mentioned above, the geodesic distance to the boundary on 
the pseudosphere is infinite. Therefore, the two-point function in the LHS of (15) can be factorized into a product of two one-point functions and satisfies

$$
B_{-}^{(N)}(\alpha)=U^{N}(\alpha) U^{R}(-b / 2) .
$$

Combining all these and using (27), we obtain the following nonlinear functional equation in the $\eta \rightarrow 1$ limit:

$$
\frac{\Gamma\left(\frac{1-b^{2}}{2}\right) U^{N}(\alpha) U^{R}\left(-\frac{b}{2}\right)}{\Gamma\left(-b^{2}\right) \Gamma\left(\alpha b-\frac{b^{2}}{2}+\frac{1}{2}\right)}=\frac{U^{R}\left(\alpha-\frac{b}{2}\right)}{\Gamma\left(\alpha b-b^{2}\right)}+\frac{\pi \mu b^{2} U^{R}\left(\alpha+\frac{b}{2}\right)}{\gamma\left(\frac{1-b^{2}}{2}\right) \Gamma(\alpha b)\left(\alpha b-\frac{b^{2}}{2}-\frac{1}{2}\right)} .
$$

Analysis of the other two-point function

$$
G_{\alpha,-b / 2}^{R}\left(\xi, \xi^{\prime}\right)=\left\langle R_{\alpha}(\xi) R_{-b / 2}\left(\xi^{\prime}\right)\right\rangle
$$

goes along the same line and leads to the second functional equation

$$
\frac{\Gamma\left(\frac{1-b^{2}}{2}\right) U^{R}(\alpha) U^{R}\left(-\frac{b}{2}\right)}{\Gamma\left(-b^{2}\right) \Gamma\left(\alpha b-\frac{b^{2}}{2}\right)}=\frac{U^{N}\left(\alpha-\frac{b}{2}\right)}{\Gamma\left(\alpha b-b^{2}-\frac{1}{2}\right)}+\frac{\pi \mu b^{2} U^{N}\left(\alpha+\frac{b}{2}\right)}{\gamma\left(\frac{1-b^{2}}{2}\right) \Gamma\left(\alpha b+\frac{1}{2}\right)} .
$$

Here, we used the following $(\mathrm{R})$-sector conformal blocks 14

$$
\begin{aligned}
& \mathcal{F}_{+}^{R}(\eta)=\eta^{\frac{\alpha b}{2}+\frac{1}{8}}(1-\eta)^{-\frac{b^{2}}{4}+\frac{3}{8}}\left(1+\sqrt{1-\frac{1}{\eta}}\right)^{1 / 2} F\left(\alpha b-b^{2}-\frac{1}{2}, \frac{1-b^{2}}{2} ; \alpha b-\frac{b^{2}}{2} ; \eta\right) \\
& \mathcal{F}_{-}^{R}(\eta)=\eta^{\frac{b^{2}}{2}-\frac{\alpha b}{2}-\frac{5}{8}}(1-\eta)^{-\frac{b^{2}}{4}-\frac{1}{8}}\left(1-\sqrt{1-\frac{1}{\eta}}\right)^{1 / 2} F\left(\frac{-1-b^{2}}{2}, \frac{1}{2}-\alpha b ;-\alpha b+\frac{b^{2}}{2}+1 ; \eta\right) .
\end{aligned}
$$

It is worth mentioning on the superindices $(\epsilon)$ we have omitted in the correlation function (34). When one (R) field has $\epsilon=+$ and the other $\epsilon=-$, the two-point function becomes proportional to $\left\langle\sigma^{(+)} \sigma^{(-)}\right\rangle$or $\langle\psi\rangle$. And the fermion one-point function should vanish due to the Lorenz invariance. If both $(\mathrm{R})$ fields have the same index, either $\epsilon=+$ or - , the previous derivation holds as it is and the same functional relations are obtained.

The SLFT satisfies the duality $b \rightarrow 1 / b$. This property requires considering another degenerate $(\mathrm{R})$ operator $R_{-1 / 2 b}$ which generates two more functional equations in addition to Eqs.(33) and (35). These additional equations can be obtained by just replacing the coupling constant $b$ with $1 / b$ and the paramter $\mu$ by the "dual" $\widetilde{\mu}$ satisfying

$$
\pi \widetilde{\mu} \gamma\left(\frac{Q}{2 b}\right)=\left[\pi \mu \gamma\left(\frac{b Q}{2}\right)\right]^{1 / b^{2}}
$$

Therefore, the one-point functions $U^{N}(\alpha)$ and $U^{R}(\alpha)$ should satisfy four nonlinear functional equations. 
We have found the solutions to these overdetermined nonlinear equations as follows:

$$
\begin{aligned}
U_{m n}^{N}(\alpha) & =\frac{\sin \left(\frac{\pi Q}{2 b}\right) \sin \left(\frac{\pi b Q}{2}\right) \sin \left[m \pi\left(\frac{Q}{2 b}-\frac{\alpha}{b}\right)\right] \sin \left[n \pi\left(\frac{b Q}{2}-b \alpha\right)\right]}{\sin \left(\frac{m \pi Q}{2 b}\right) \sin \left(\frac{n \pi b Q}{2}\right) \sin \left[\pi\left(\frac{Q}{2 b}-\frac{\alpha}{b}\right)\right] \sin \left[\pi\left(\frac{b Q}{2}-b \alpha\right)\right]} U_{11}^{N}(\alpha) \\
U_{m n}^{R}(\alpha) & =\frac{\sin \left(\frac{\pi Q}{2 b}\right) \sin \left(\frac{\pi b Q}{2}\right) \sin \left[m \pi\left(\frac{Q}{2 b}-\frac{\alpha}{b}+\frac{1}{2}\right)\right] \sin \left[n \pi\left(\frac{b Q}{2}-b \alpha+\frac{1}{2}\right)\right]}{\sin \left(\frac{m \pi Q}{2 b}\right) \sin \left(\frac{n \pi b Q}{2}\right) \cos \left[\pi\left(\frac{Q}{2 b}-\frac{\alpha}{b}\right)\right] \cos \left[\pi\left(\frac{b Q}{2}-b \alpha\right)\right]} U_{11}^{R}(\alpha)(38
\end{aligned}
$$

where the 'basic' solutions are given by

$$
\begin{aligned}
& U_{11}^{N}(\alpha)=\left[\pi \mu \gamma\left(\frac{b Q}{2}\right)\right]^{-\alpha / b} \frac{\Gamma\left(\frac{b Q}{2}\right) \Gamma\left(\frac{Q}{2 b}\right) \frac{Q}{2}}{\Gamma\left(-\alpha b+\frac{b Q}{2}\right) \Gamma\left(-\frac{\alpha}{b}+\frac{Q}{2 b}\right)\left(\frac{Q}{2}-\alpha\right)} \\
& U_{11}^{R}(\alpha)=\left[\pi \mu \gamma\left(\frac{b Q}{2}\right)\right]^{-\alpha / b} \frac{\Gamma\left(\frac{b Q}{2}\right) \Gamma\left(\frac{Q}{2 b}\right) \frac{Q}{2}}{\Gamma\left(-\alpha b+\frac{b Q}{2}+\frac{1}{2}\right) \Gamma\left(-\frac{\alpha}{b}+\frac{Q}{2 b}+\frac{1}{2}\right)} .
\end{aligned}
$$

This is our main result in this section. There are infinite number of possible solutions which are parametrized by two integers $(m, n)$. For these to be solutions, we find that the two integers should satisfy $m-n=$ even. The basic solutions, Eqs.(39) and (40), can be interpreted as the one-point functions of the bulk operators $N_{\alpha}$ and $R_{\alpha}$ corresponding to the vacuum $\mathrm{BC}$, the $\mathrm{BC}$ corresponding to the bulk vacuum operator $N_{0}$. Then, the general solutions, Eqs.(37) and (38), can be identified with the one-point functions with the conformal BC $(m, n)$ classified by Cardy [6]. We will discuss more about this issue in sect.4. Since $m-n=$ even, the one-point functions we obtained correspond to the (NS)-type BCs only. This seems consistent with the fact that only the (NS) boundary operators arise when the (NS) or (R) bulk degenerate operators approach the boundary corresponding to the vacuum $\mathrm{BC}$.

We also note that Eqs.(37) and (38) satisfy so-called bulk "reflection relations":

$$
U_{m, n}^{N}(\alpha)=D^{(N)}(\alpha) U_{m, n}^{N}(Q-\alpha), \quad U_{m, n}^{R}(\alpha)=D^{(R)}(\alpha) U_{m, n}^{R}(Q-\alpha)
$$

where $D^{(N)}(\alpha)$ and $D^{(R)}(\alpha)$ are the (NS) and the (R) reflection amplitudes derived in [4, 5]. One can also notice that new functions defined by

$$
\widetilde{U}_{m, n}^{N}=U_{m, n}^{N}, \quad \widetilde{U}_{m, n}^{R}=-U_{m, n}^{R}
$$

become again solutions to the above nonlinear equations. We will discuss the meaning of the solutions from the viewpoint of the generalized Cardy formalism later.

Finally, we consider a special case where $\alpha=0$. From Eq.(37), one can notice that one-point function of the identity operator is normalized in such a way that $U^{N}(0)=1$. Then, one-point function of the spin field with $(m, n) \mathrm{BC}$ is given by Eq.(38)

$$
\left\langle\sigma^{( \pm)}\right\rangle_{(m, n)}=U_{m n}^{R}(0)
$$




\section{Bulk One-Point Function with Boundary}

In this section, we define the SLFT on half plane where superconformally invariant boundary action is imposed. We choose the following boundary action at $y=0$

$$
\mathcal{L}_{B}=\frac{\mu_{B}}{2} e^{b \phi / 2} a(\psi-i \gamma \bar{\psi})(x)
$$

with $\gamma= \pm 1$ and the fermionic zero-mode $a$ satisfying 15

$$
\sigma^{( \pm)}=a \sigma^{(\mp)} \quad \text { and } \quad a^{2}=1
$$

This action includes additional boundary parameter $\mu_{B}$ which generates continuous family of the BCs. The boundary equations of motion are given by

$$
\begin{aligned}
\frac{1}{2 \pi} \partial_{y} \phi & =-\frac{1}{2} b \mu_{B} a(\psi-i \gamma \bar{\psi}) e^{b \phi / 2} \\
\frac{i}{2 \pi} \psi & =\mu_{B} e^{b \phi / 2} a, \quad \frac{i}{2 \pi} \bar{\psi}=i \gamma \mu_{B} e^{b \phi / 2} a
\end{aligned}
$$

which lead to

$$
(\psi+i \gamma \bar{\psi})=0
$$

Notice that Eq.(48) is the well-known fermion BC imposed by Cardy. Plugging these constraints back into the action, one can simplify the boundary action

$$
\mathcal{L}_{B}=\mu_{B} e^{b \phi / 2} a \psi
$$

This term is different from that preserving boundary integrability such as considered in [16]. Main difference is that our action is preserving not only integrability but also superconformal symmetry. Indeed, the boundary action is nothing but the screening operator which guarantees the symmetry.

One can see that physical quantities should contain only even powers of $\mu_{B}$ because of the fermionic zero-mode. While the bulk properties of the boundary SLFT should be identical, we should define the boundary operators. As in the bulk, there are two sectors, the (NS) and (R) boundary operators

$$
n_{\beta}=e^{\beta \phi / 2}(x), \quad r_{\beta}=\sigma^{(\epsilon)} e^{\beta \phi / 2}(x) .
$$

The procedure to derive the functional equations satisfied by the bulk one-point functions are identical to that in sect.2. Major difference arises when the bulk degenerate operator $R_{-b / 2}$ approaches the boundary as $z \rightarrow \bar{z}$. The LHS of Eq.(22) can be evaluated by the boundary OPE which generates the boundary operator $n_{0}$ and $n_{-b}$. We choose the identity operator $n_{0}$, or the boundary vacuum state, since we are interested in the bulk 
one-point function. The fusion of the degenerate field $R_{-b / 2}$ can be computed by a first order perturbation from the boundary action:

$$
\begin{aligned}
\mathcal{R}^{(\epsilon)}(-b / 2, Q) & =-\mu_{B} \int d x\left\langle R_{-b / 2}^{(\epsilon)}\left(\frac{i}{2}\right) a \psi(x) e^{b \phi_{B} / 2}(x) e^{Q \phi_{B} / 2}(\infty)\right\rangle \\
& =\mu_{B} \int d x|x-i / 2|^{b^{2}-1}=2 \pi \mu_{B} \frac{\Gamma\left(-b^{2}\right)}{\Gamma\left(\frac{1-b^{2}}{2}\right)^{2}} .
\end{aligned}
$$

Here, we used the formula

$$
\langle\sigma(\xi, \bar{\xi}) a \psi(x)\rangle=\frac{-1}{|x-\xi|^{1 / 2}|\xi-\bar{\xi}|^{3 / 8}} .
$$

Again, the dependence on the superindex $\epsilon$ disappears so that we can suppress it. With the vacuum state on the boundary, the two-point function becomes the bulk one-point function of the operator $N_{\alpha}$. Equating this with the RHS of Eq.(22) gives the functional equation

$$
\frac{2 \pi \mu_{B}}{\Gamma\left(\frac{1-b^{2}}{2}\right)} U^{N}(\alpha)=\frac{\Gamma\left(\alpha b-\frac{b^{2}}{2}+\frac{1}{2}\right)}{\Gamma\left(\alpha b-b^{2}\right)} U^{R}\left(\alpha-\frac{b}{2}\right)+\frac{\pi \mu b^{2} \Gamma\left(\alpha b-\frac{b^{2}}{2}-\frac{1}{2}\right)}{\gamma\left(\frac{1-b^{2}}{2}\right) \Gamma(\alpha b)} U^{R}\left(\alpha+\frac{b}{2}\right) .
$$

Similar consideration for the $G_{\alpha,-b / 2}^{R}$ leads to

$$
\frac{2 \pi \mu_{B}}{\Gamma\left(\frac{1-b^{2}}{2}\right)} U^{R}(\alpha)=\frac{\Gamma\left(\alpha b-\frac{b^{2}}{2}\right)}{\Gamma\left(\alpha b-b^{2}-\frac{1}{2}\right)} U^{N}\left(\alpha-\frac{b}{2}\right)+\frac{\pi \mu b^{2} \Gamma\left(\alpha b-\frac{b^{2}}{2}\right)}{\gamma\left(\frac{1-b^{2}}{2}\right) \Gamma\left(\alpha b+\frac{1}{2}\right)} U^{N}\left(\alpha+\frac{b}{2}\right) .
$$

As before, one should consider the dual equations coming from the dual degenerate operator $R_{-1 / 2 b}$.

The solutions of Eqs.(53) and (54) can be found as

$$
\begin{gathered}
U^{N}(\alpha)=\mathcal{N} b\left[\pi \mu \gamma\left(\frac{b Q}{2}\right)\right]^{\frac{Q-2 \alpha}{2 b}} \Gamma\left(\left(\alpha-\frac{Q}{2}\right) b\right) \Gamma\left(1+\left(\alpha-\frac{Q}{2}\right) \frac{1}{b}\right) \cosh \left[\left(\alpha-\frac{Q}{2}\right) \pi s\right] \\
U^{R}(\alpha)=\mathcal{N}\left[\pi \mu \gamma\left(\frac{b Q}{2}\right)\right]^{\frac{Q-2 \alpha}{2 b}} \Gamma\left(\left(\alpha-\frac{b}{2}\right) b\right) \Gamma\left(\left(\alpha-\frac{1}{2 b}\right) \frac{1}{b}\right) \cosh \left[\left(\alpha-\frac{Q}{2}\right) \pi s\right],
\end{gathered}
$$

where the normalization factor $\mathcal{N}$ is given by

$$
\mathcal{N}=\left[\pi \mu \gamma\left(\frac{b Q}{2}\right)\right]^{-Q / 2 b}[b \Gamma(-Q b / 2) \Gamma(1-Q / 2 b) \cosh (Q \pi s / 2)]^{-1}
$$

so that $U^{N}(0)=1$. Here, the boundary parameter $s$ is related to $\mu_{B}$ by

$$
\frac{\mu_{B}^{2}}{\mu b^{2}} \sin \left(\frac{\pi b Q}{2}\right)=\cosh ^{2}\left(\frac{\pi b s}{2}\right) .
$$


It is possible to find another conformal BC by changing $a \rightarrow-a$. This introduces extra '-' sign in the LHS of the functional equations, Eqs.(53) and (54), so that the solutions are found to be

$$
\widetilde{U}^{(N)}=U^{(N)}, \quad \widetilde{U}^{(R)}=-U^{(R)} .
$$

The solutions Eqs.(55) and (56) are our main result in this section. Notice that these are self-dual if the parameter $s$ is invariant and $\mu \rightarrow \widetilde{\mu}$ as Eq.(36). The continuous parameter $s$ coming from $\mu_{B}$ generates a continuous family of conformally invariant BCs. We will discuss how these BCs can be consistent with the generalized Cardy formalism in the next section. One can also check that these satisfy the bulk reflection relations Eq.(41).

The one-point function can be checked by a perturbative analysis. Defining the third and fourth terms in Eq.(11) as $V_{b}$ and the boundary action in Eq.(44) as $B_{b}$, one can express an one-point function as an infinite sum of these perturbation terms,

$$
\left\langle O_{\alpha}(\xi, \bar{\xi})\right\rangle=\sum_{p, q} \frac{1}{p ! q !}\left\langle O_{\alpha}(\xi, \bar{\xi}) V_{b}^{p} B_{b}^{q}\right\rangle_{0}
$$

where the evaluation is made with $\mu=\mu_{B}=0$. It is well known that the perturbative results are non-vanishing only at the on-shell condition $\alpha=Q / 2-(p+q / 2) b$ and correspond to the residue of the one-point function as follows:

$$
\begin{aligned}
\text { residue }\left.U^{N}(\alpha)\right|_{\alpha=Q / 2-(p+q / 2) b} & =\mathcal{N}\left\langle e^{\alpha \phi}(i / 2)\right\rangle \\
\text { residue }\left.U^{R}(\alpha)\right|_{\alpha=Q / 2-(p+q / 2) b} & =\mathcal{N}\left\langle\sigma e^{\alpha \phi}(i / 2)\right\rangle,
\end{aligned}
$$

where $p$ is a non-negative integer and $q$ is a non-negative even integer for the (NS)-sector and odd for the (R)-sector. From Eqs.(55) and (56), one can find nontrivial pole structure of $U^{N}$ at $p+q / 2=0$,

$$
U^{N}(\alpha) \cong \frac{\mathcal{N}}{\alpha-\frac{Q}{2}},
$$

and at $p+q / 2=1$,

$$
U^{N}(\alpha) \cong \pi \mu b^{2} \gamma\left(\frac{b Q}{2}\right) \Gamma\left(-b^{2}\right) \cosh (\pi b s) \frac{\mathcal{N}}{\alpha-\frac{Q}{2}+b} .
$$

On the other hand, the perturbative calculation for $p+q / 2=0$ gives

$$
\left\langle e^{\alpha \phi}(i / 2,-i / 2)\right\rangle_{0}=1 \text {. }
$$

and for $p+q / 2=1$

$$
\begin{aligned}
\left\langle e^{\alpha \phi}(i / 2,-i / 2) V_{b}\right\rangle_{0} & =\mu b^{2} \int_{\operatorname{Im} z>0} d^{2} \xi|\xi-i / 2|^{-2 \alpha b}|\xi+i / 2|^{-2 \alpha b}|\xi-\bar{\xi}|^{-b Q} \\
& =(\pi b Q) \mu b^{2} \Gamma(-b Q) \gamma\left(\frac{b Q}{2}\right)
\end{aligned}
$$




$$
\begin{aligned}
\left\langle e^{\alpha \phi}(i / 2,-i / 2) B_{b}^{2}\right\rangle_{0} & =-\mu_{B}^{2} \int_{-\infty}^{\infty} d x_{1} d x_{2}\left|x_{1}-i / 2\right|^{-2 \alpha b}\left|x_{2}-i / 2\right|^{-2 \alpha b}\left|x_{1}-x_{2}\right|^{-b Q} \\
& =-\mu_{B}^{2}(4 \pi b Q) \Gamma(-b Q) \gamma\left(\frac{\pi b Q}{2}\right) \sin \left(\frac{b Q}{2}\right)
\end{aligned}
$$

Combining the two contributions gives the correct residue of the one-point function.

The first non-trivial check for the $(\mathrm{R})$-sector arises at $p+q / 2=1 / 2$

$$
U^{R}(\alpha) \cong \frac{\mu_{B} \pi}{\alpha-\frac{1}{2 b}}
$$

This is consistent with the perturbative result

$$
\left\langle\sigma e^{\alpha \phi}(i / 2,-i / 2) B_{b}\right\rangle_{0}=\mu_{B} \pi
$$

\section{Boundary states for the super-LFT}

For the super-CFTs, Virasoro characters are defined for the (NS) sector, the (R)-sector, and the (NS)-sector. The characters of the primary states for the generic value of $P$, which have no null-states, are given by [17]:

$$
\begin{aligned}
\chi_{P}^{N S}\left(q^{2}\right) & =\sqrt{\frac{\theta_{3}(q)}{\eta(q)}} \frac{q^{P^{2} / 2}}{\eta(q)} \\
\chi_{P}^{\widetilde{N S}}(q) & =\sqrt{\frac{\theta_{4}(q)}{\eta(q)}} \frac{q^{P^{2} / 2}}{\eta(q)} \\
\chi_{P}^{R}(q) & =\sqrt{\frac{\theta_{2}(q)}{2 \eta(q)}} \frac{q^{P^{2} / 2}}{\eta(q)}
\end{aligned}
$$

where $q=\exp (2 \pi i \tau)$. Under the modular transformation $\tau \rightarrow \tau^{\prime}=-1 / \tau$, the characters transform

$$
\begin{aligned}
\chi_{P}^{N S}(\tau) & =\int_{-\infty}^{\infty} d P^{\prime} e^{-2 \pi i P P^{\prime}} \chi_{P^{\prime}}^{N S}\left(\tau^{\prime}\right) \\
\chi_{P}^{\widetilde{N S}}(\tau) & =\int_{-\infty}^{\infty} d P^{\prime} e^{-2 \pi i P P^{\prime}} \chi_{P^{\prime}}^{R}\left(\tau^{\prime}\right) \\
\chi_{P}^{R}(\tau) & =\int_{-\infty}^{\infty} d P^{\prime} e^{-2 \pi i P P^{\prime}} \chi_{P^{\prime}}^{\widetilde{N S}}\left(\tau^{\prime}\right) .
\end{aligned}
$$

On the other hand, the modular transformations of the characters for the (NS) degenerate fields in Eq.(12) are given by

$$
\begin{aligned}
& \chi_{m, n}^{N S}(q)=\int_{-\infty}^{\infty} d P \chi_{P}^{N S}\left(q^{\prime}\right) 2 \sinh (\pi m P / b) \sinh (\pi m P b) \\
& \chi_{m, n}^{\widetilde{N S}}(q)=\int_{-\infty}^{\infty} d P \chi_{P}^{R}\left(q^{\prime}\right) \begin{cases}2 \sinh (\pi m P / b) \sinh (\pi m P b) & m, n=\text { even } \\
2 \cosh (\pi m P / b) \cosh (\pi m P b) & m, n=\text { odd } .\end{cases}
\end{aligned}
$$


According to Cardy's formalism, one can associate a conformal BC with each primary state [6]. Since the SLFT is an irrational CFT with infinite number of primary states, there will be infinite number of conformal BCs. One can classify these into 'discrete BCs' and 'continuous BCs' for the degenerate and non-degenerate primary states, respectively. It is natural to start with the discrete BCs $(m, n)$. With $m-n=$ even, there are the (NS)-type BCs corresponding to the (NS) degenerate fields $N_{\alpha_{m, n}}$. We will denote the corresponding boundary states by $|(m, n)\rangle$. Due to the super conformal symmetry, one needs to introduce additional BCs $(\widetilde{m, n})$ and corresponding boundary states $|(\widetilde{m, n})\rangle[12]$. Let us consider $(m, n)$ BCs first. Through the modular transformation, one can obtain

$$
\chi_{m, n}^{N S}(\tau)=\int_{-\infty}^{\infty} d P \Psi_{m, n}^{N S}(P) \Psi_{1,1}^{N S}(P)^{\dagger} \chi_{P}^{N S}\left(\tau^{\prime}\right)
$$

where the amplitude is defined by

$$
\left.\Psi_{m, n}^{N S}(P)=\langle(m, n) \mid \alpha, \mathrm{NS}\rangle\right\rangle
$$

where $|\alpha, N S\rangle\rangle$ is the (NS) Ishibashi state with $\alpha=Q / 2+i P$.

Using Eq.(75) into Eq.(77), one can obtain a relation

$$
\Psi_{1,1}^{N S}(P)^{\dagger} \Psi_{m, n}^{N S}(P)=2 \sinh (\pi m P / b) \sinh (\pi m P b) .
$$

We can set the basic amplitude for $m=n=1$ as

$$
\Psi_{1,1}^{N S}(P)=\frac{\pi \sqrt{2}}{P \Gamma(-i P b) \Gamma(-i P / b)}\left[\pi \mu \gamma\left(\frac{b Q}{2}\right)\right]^{-i P / b} .
$$

Then, one can obtain the relation

$$
\Psi_{m, n}^{N S}(P)=\Psi_{1,1}^{N S}(P) \frac{\sinh \left(\frac{m \pi P}{b}\right) \sinh (n \pi P b)}{\sinh \left(\frac{\pi P}{b}\right) \sinh (\pi P b)}
$$

by the Cardy formula.

It has been shown in [10] that the amplitudes are related to the one-point functions by

$$
U_{\tilde{k}}(\phi)=\frac{\langle\tilde{k} \mid \phi\rangle\rangle}{\langle\tilde{k} \mid \mathbf{1}\rangle\rangle} .
$$

For the SLFT, this formula becomes

$$
U_{m, n}^{N}(\alpha)=\frac{\langle(m, n) \mid \alpha, \mathrm{NS}\rangle\rangle}{\langle(m, n) \mid 0, \mathrm{NS}\rangle\rangle}
$$


From this and Eq.(80), one can obtain

$\frac{U_{m, n}^{N}(\alpha)}{U_{1,1}^{N}(\alpha)}=\frac{\langle(m, n) \mid \alpha, \mathrm{NS}\rangle\rangle}{\langle(m, n) \mid 0, \mathrm{NS}\rangle\rangle} \frac{\langle(1,1) \mid 0, \mathrm{NS}\rangle\rangle}{\langle(1,1) \mid \alpha, \mathrm{NS}\rangle\rangle}=\frac{\sinh \left(\frac{m \pi P}{b}\right) \sinh (n \pi P b)}{\sinh \left(\frac{\pi P}{b}\right) \sinh (\pi P b)} \frac{\sin \left(\frac{\pi Q}{2 b}\right) \sin \left(\frac{\pi Q b}{2}\right)}{\sin \left(\frac{m \pi Q}{2 b}\right) \sin \left(\frac{n \pi Q b}{2}\right)}$.

This result is identical to Eq.(37), which shows that the one-point functions obtained from the functional relations are consistent with the modular transformation properties.

Now let us consider the partition function on a strip with $(m, n)$ and $\left(m^{\prime}, n^{\prime}\right)$ BCs on both boundaries. Using the fusion procedure, one can obtain

$$
\begin{aligned}
Z_{(m, n),\left(m^{\prime}, n^{\prime}\right)}^{N S}(\tau) & =\int_{-\infty}^{\infty} d P \chi_{P}^{N S}\left(\tau^{\prime}\right) \Psi_{m, n}^{N S}(P) \Psi_{m^{\prime}, n^{\prime}}^{N S}(P)^{\dagger} \\
& =\int_{-\infty}^{\infty} d P \chi_{P}^{N S}\left(\tau^{\prime}\right) \frac{2 \sinh (m \pi P / b) \sinh (n \pi P b) \sinh \left(m^{\prime} \pi P / b\right) \sinh \left(n^{\prime} \pi P b\right)}{\sinh (\pi P / b) \sinh (\pi P b)} \\
& =\sum_{k=0}^{\min \left(m, m^{\prime}\right)-1} \sum_{l=0}^{\min \left(n, n^{\prime}\right)-1} \chi_{\left(m+m^{\prime}-1-2 k\right),\left(n+n^{\prime}-1-2 l\right)}^{N S}(\tau)
\end{aligned}
$$

in accordance with the fusion algebra. The character for the non-degenerate case with $P=s / 2$ satisfies

$$
\chi_{s}^{N S}(\tau)=\int_{-\infty}^{\infty} d P \chi_{P}^{N S}\left(\tau^{\prime}\right) \cos (\pi s P)=\int_{-\infty}^{\infty} d P \chi_{P}^{N S}\left(\tau^{\prime}\right) \Psi_{s}^{N S}(P) \Psi_{1,1}^{N S}(P)^{\dagger},
$$

with $\left.\Psi_{s}^{N S}(P)=\langle\tilde{s} \mid P, N S\rangle\right\rangle$.

From this, one can find the amplitudes for a general non-degenerate (NS) boundary state satisfy

$$
\Psi_{s}^{N S}(P)=\Psi_{1,1}^{N S}(P) \frac{\cos (\pi s P)}{2 \sinh \left(\frac{\pi P}{b}\right) \sinh (\pi P b)} .
$$

Again, from Eq.(81), the amplitudes are related to the one-point functions by

$$
\frac{U^{N}(\alpha)}{U_{1,1}^{N}(\alpha)}=\frac{\langle\tilde{s} \mid \alpha, \mathrm{NS}\rangle\rangle}{\langle\tilde{s} \mid 0, \mathrm{NS}\rangle\rangle} \frac{\langle(1,1) \mid 0, \mathrm{NS}\rangle\rangle}{\langle(1,1) \mid \alpha, \mathrm{NS}\rangle\rangle}=\frac{\cos (\pi P s)}{\cosh \left(\frac{Q \pi s}{2}\right)} \frac{\sin (\pi Q / 2 b) \sin (\pi Q b / 2)}{\sinh (\pi P b) \sinh (\pi P / b)} .
$$

This can be checked to be correct by Eqs. (39) and (55).

The partition function with a discrete $\mathrm{BC}$ on one side and a continuous $\mathrm{BC}$ on the other is given as follows:

$$
\begin{aligned}
Z_{(m, n), s}^{N S}(\tau) & =\int_{-\infty}^{\infty} d P \chi_{P}^{N S}\left(\tau^{\prime}\right) \Psi_{m, n}^{N S}(P) \Psi_{s}^{N S}(P)^{\dagger} \\
& =\int_{-\infty}^{\infty} d P \chi_{P}^{N S}\left(\tau^{\prime}\right) \frac{\sinh (m \pi P / b) \sinh (n \pi P b)}{\sinh (\pi P / b) \sinh (\pi P b)} \cos (\pi s P) \\
& =\sum_{k=0}^{m-1} \sum_{l=0}^{n-1} \chi_{s+i(m-1-2 k) / b+i(n-1-2 l) b}^{N S}(\tau)
\end{aligned}
$$


which goes with the fusion algebra.

The partition function $Z_{s, s^{\prime}}^{N S}(\tau)$ with contiuous BCs on both boundaries, $s$ and $s^{\prime}$, is given as

$$
Z_{s, s^{\prime}}^{N S}(\tau)=\int_{-\infty}^{\infty} d P \chi_{P}^{N S}\left(\tau^{\prime}\right) \Psi_{s}^{N S}(P) \Psi_{s^{\prime}}^{N S}(P)^{\dagger}
$$

This can be rewritten as

$$
\begin{aligned}
Z_{s, s^{\prime}}^{N S}(\tau) & =\int_{-\infty}^{\infty} d P^{\prime} \int_{-\infty}^{\infty} d P e^{-2 i \pi P P^{\prime}} \chi_{P}^{N S}(\tau) \Psi_{s}^{N S}\left(P^{\prime}\right) \Psi_{s^{\prime}}^{N S}\left(P^{\prime}\right)^{\dagger} \\
& =\int_{0}^{\infty} d P \chi_{P}^{N S}(\tau) \rho_{s s^{\prime}}^{N S}(P),
\end{aligned}
$$

where $\rho_{s s^{\prime}}^{N S}(P)$ is the density of states,

$$
\rho_{s s^{\prime}}^{N S}(P)=\int_{-\infty}^{\infty} \frac{d t}{\pi} e^{-2 i P t} \frac{\cos (s t) \cos \left(s^{\prime} t\right)}{\sinh (t / b) \sinh (t b)} .
$$

This quantity is not well-defined at $P=0$ and is to be properly regularized. This density of states is, on the other hand, conjectured to be related with the boundary two-point function of $n_{\beta}^{s s^{\prime}}$ with $\beta=Q / 2+i P, d_{B}^{N S}\left(P \mid s, s^{\prime}\right)$, by

$$
\rho_{s, s^{\prime}}^{N S}(P)=-\frac{i}{2 \pi} \frac{d}{d P} \log d_{B}^{N S}\left(P \mid s, s^{\prime}\right) .
$$

It is remarkable that this relation is identical to that of the LFT. This means that the boundary (NS) two-point functions have the same dependence on the boundary parameters as the LFT which is obtained in 87 .

Now we consider the $(\mathrm{R})$ operator propagating in the strip. From the relation

$$
\chi_{m, n}^{\widetilde{N S}}(\tau)=\int_{-\infty}^{\infty} d P \Psi_{m, n}^{R}(P) \Psi_{1,1}^{R}(P)^{\dagger} \chi_{P}^{R}\left(\tau^{\prime}\right),
$$

one can obtain

$$
\Psi_{1,1}^{R}(P)^{\dagger} \Psi_{m, n}^{R}(P)=\left\{\begin{array}{lr}
2 \sinh (\pi m P / b) \sinh (\pi m P b) & m, n=\text { even } \\
2 \cosh (\pi m P / b) \cosh (\pi m P b) & m, n=\text { odd } .
\end{array}\right.
$$

Here, we define the amplitude $\left.\Psi_{m, n}^{R}(P)=\langle(m, n) \mid \alpha, R\rangle\right\rangle$ as before.

These amplitudes are related to the one-point fuctions by

$$
U_{m, n}^{R}(\alpha)=\frac{\langle(m, n) \mid \alpha, R\rangle\rangle}{\langle(m, n) \mid 0, R\rangle\rangle},
$$

and satisfy

$$
\frac{U_{m, n}^{R}(\alpha)}{U_{1,1}^{R}(\alpha)}=\frac{\langle(m, n) \mid \alpha, R\rangle\rangle}{\langle(m, n) \mid 0, R\rangle\rangle} \frac{\langle(1,1) \mid 0, R\rangle\rangle}{\langle(1,1) \mid \alpha, R\rangle\rangle} .
$$


One can check that this relation is consistent with Eqs.(94) and (38). This shows again that the functional relations are consistent with the modular transformation.

The partition function with $(m, n)$ and $\left(m^{\prime}, n^{\prime}\right)$ BCs is written as

$$
\begin{gathered}
Z_{(m, n),\left(m^{\prime}, n^{\prime}\right)}^{\widetilde{N S}}(\tau)=\int_{-\infty}^{\infty} d P \sqrt{2} \chi_{P}^{R}\left(\tau^{\prime}\right) \cdot \Psi_{m, n}^{R}(P) \Psi_{m^{\prime}, n^{\prime}}^{R}(P)^{\dagger} \\
=\sum_{k=0}^{\min \left(m, m^{\prime}\right)-1} \sum_{l=0}^{\min \left(n, n^{\prime}\right)-1} \chi_{\left(m+m^{\prime}-1-2 k\right),\left(n+n^{\prime}-1-2 l\right)}^{\widetilde{N S}}(\tau)
\end{gathered}
$$

and is consistent with fusion algebra.

The character for the continuous boundary parameter $s$ satisfies

$$
\chi_{s}^{\widetilde{N S}}(\tau)=\int_{-\infty}^{\infty} d P \chi_{P}^{R}\left(\tau^{\prime}\right) \cos (\pi s P)=\int_{-\infty}^{\infty} d P \chi_{P}^{R}\left(\tau^{\prime}\right) \Psi_{s}^{R}(P) \Psi_{1,1}^{R}(P)^{\dagger} .
$$

The (R) amplitude for a general boundary parameter $s$ is given as

$$
\Psi_{s}^{R}(P)=\Psi_{1,1}^{R}(P) \frac{\cos (\pi s P)}{2 \cosh \left(\frac{\pi P}{b}\right) \cosh (\pi P b)} .
$$

Again, from Eq.(81), the amplitudes are related to the one-point functions by

$$
\frac{U^{R}(\alpha)}{U_{1,1}^{R}(\alpha)}=\frac{\langle\tilde{s} \mid \alpha, R\rangle\rangle}{\langle\tilde{s} \mid 0, R\rangle\rangle} \frac{\langle(1,1) \mid 0, R\rangle\rangle}{\langle(1,1) \mid \alpha, R\rangle\rangle}=\frac{\cos (\pi P s)}{\cosh \left(\frac{Q \pi s}{2}\right)} \frac{\cos (\pi Q / 2 b) \cos (\pi Q b / 2)}{\cosh (\pi P b) \cosh (\pi P / b)} .
$$

This can be checked to be correct by Eqs.(40) and (56).

The partition function with mixed BCs is given as follows:

$$
\begin{aligned}
Z_{(m, n), s}^{\widetilde{N S}}(\tau) & =\int_{-\infty}^{\infty} d P \chi_{P}^{R}\left(\tau^{\prime}\right) \Psi_{m, n}^{R}(P) \Psi_{s}^{R}(P)^{\dagger} \\
& =\sum_{k=0}^{m-1} \sum_{l=0}^{n-1} \chi_{s+i(m-1-2 k) / b+i(n-1-2 l) b}^{\widetilde{N S}}(\tau)
\end{aligned}
$$

One can consider $(\widetilde{m, n})$ BCs in the same way and can associate the amplitudes $\Psi \frac{N S}{m, n}$ and $\Psi \frac{R}{m, n}$ with $\tilde{U}_{m, n}^{N}$ and $\tilde{U}_{m, n}^{R}$, respectively. From Eq.(42), one can see that

$$
\Psi_{m, n}^{N S}=\Psi \frac{N S}{m, n}, \quad \Psi_{m, n}^{R}=-\Psi \frac{R}{m, n}
$$

Similar result holds for the continuous BCs and can be compared with Eq.(59). These results are consistent with those of the rational super-CFTs considered in [12]. 


\section{Conclusions}

In this paper, we have studied the SLFT in two-dimensional space-time with boundary applying the same method used for the LFT. However, the one-point functions of the SLFT satisfy more complicated functional relations due to the existence of two sectors, the (NS) and (R). By solving the functional relations, we find not only the one-point functions but also the relation between the parameter $\mu_{B}$ in the boundary action and that for the boundary condition.

We have also related the one-point functions in the pseudosphere to the conformal BCs and showed that they are consistent with the Cardy formalism if one takes care of the peculiar aspects of the super-CFTs in the same way as the rational cases [11, 12]. Then, this result has been used to understand boundary two-point functions for the SLFT with the boundary action. We conclude that the boundary (NS) two-point functions have the same dependence on the boundary parameters as the LFT while explicit expression of this quantity needs more work. We hope to present it in another publication.

There are still other problems which should be further explored. The solutions of Eqs.(37) and (38) are possible only for $m-n=$ even. The functional equations for the other case may also exist. This becomes necessary when one relates to the conformal BCs. In this paper, we considered only the (NS)-type BCs since the (R)-type BCs should be associated with $m-n=$ odd.

Based on the successful results of the LFT and the SLFT, it seems the approach based on the functional relation associated with some degenerate fields are quite efficient way of dealing with irrational CFTs. In this respect, it would be interesting to apply this method to such irrational CFTs as finite Toda field theories and the LFT with $N=2$ and fractional supersymmetries. Another interesting problem is to derive the boundary

reflection amplitude from the boundary two-point functions of the $N=1$ SLFT and to obtain off-critical scaling function developed in [18].

\section{Acknowledgement}

We thank V. Fateev, R. Nepomechie and Al. Zamolodchikov for valuable discussions. We thank Univ. Montpellier II, APCTP and YVRC for hospitality. This work is supported in part by KOSEF 1999-2-112-001-5, MOST-99-N6-01-01-A-5 (CA), and Eastern Europe exchange program 12-69-002 sponsored by KISTEP (MS).

\section{References}

[1] G.-L. Gervais, Comm. Math. Phys. 130 (1990) 252.

[2] J. Teschner, Phys. Lett. B363 (1995) 65. 
[3] A. B. Zamolodchikov and Al. B. Zamolodchikov, Nucl. Phys. B477 (1996) 577.

[4] R. C. Rashkov and M. Stanishkov, Phys. Lett. B380 (1996) 49.

[5] R. H. Poghossian, Nucl.Phys. B496 (1997) 451.

[6] J. Cardy, Nucl. Phys. B240 (1984) 514.

[7] P. Lee, H. Ooguri, and J. Park, "Boundary States for $\mathrm{AdS}_{2}$ branes in $\mathrm{AdS}_{3}$ ", hep-th/0112188; B. Ponsot, V. Schomerus, and J. Teschner, "Branes in the Euclidean $\mathrm{AdS}_{3} "$, hep-th/0112198.

[8] V. A. Fateev, A. B. Zamolodchikov, and Al. B. Zamolodchikov, "Boundary Liouville Field Theory I. Boundary State and Boundary Two-point Function", hep-th/0001012.

[9] A. B. Zamolodchikov and Al. B. Zamolodchikov, "Liouville Field Theory on a Pseudosphere", hep-th/0101152.

[10] J. Cardy and D. Lewellen, Phys. Lett. B259 (1991) 274.

[11] S.A. Apikyan and D.A. Sahakyan, Mod. Phys. Lett. A14 (1999) 211.

[12] R. Nepomechie, J. Phys. A34 (2001) 6509.

[13] D. Friedan, Z. Qiu, S. Shenker, Phys. Lett. B151 (1985) 37.

[14] G. Musssardo, G. Sotkov, and M. Stanishkov, Nucl.Phys. B305 (1988) 69.

[15] S. Ghoshal, A. Zamolodchikov, Int. J. Mod. Phys. A9 (1993) 3841.

[16] J. Prata, Phys. Lett. B405 (1997) 271.

[17] Y. Matsuo and S. Yahikozawa, Phys. Lett. B178 (1986) 211; D. Kastor, Nucl. Phys. B280 (1987) 304.

[18] C. Ahn, C. Kim, and C. Rim, Nucl. Phys. B556 (1999) 505; "Reflection Amplitudes of Boundary Toda Theories and Thermodynamic Bethe ansatz", hep-th/0110218. 